\title{
Synthesis of Colloidal Carbon Quantum Dots
}

\author{
Hao Zhou, ${ }^{a}$ Shi-Huan Ren, ${ }^{2}$ Yuan-Ming Zhang, ${ }^{*, b}$ and Hai-Guang Zhao, ${ }^{\star, b}$ \\ ${ }^{a}$ Department of College of Textiles \& Clothing, Qingdao University, Qingdao, Shandong 266071, China \\ ${ }^{b}$ Department of State Key Laboratory of Bio-Fibers and Eco-Textiles \& College of Physics, Qingdao University, \\ No. 308 Ningxia Road, Qingdao, Shandong 266071, China
}

Email: hgzhao@qdu.edu.cn (H.-G. Z.), jky635004277@163.com (Y.-M. Z.)

\begin{abstract}
Carbon quantum dots (C-dots) are emerging semiconductor nanomaterials consisting of earth-abundant $\mathrm{C}, \mathrm{O}$, and $\mathrm{N}$ elements. $\mathrm{C}$-dots have many advantages such as high quantum yield, good photoand chemical-stability, low-cost, low-toxicity and easy synthesis with earth-abundant precursors. In this minireview, we summarized and updated the most recent research works for the synthesis of the fluorescent and phosphorescent C-dots. In the end, we also give our owner views for the challenges and research directions for C-dots.
\end{abstract}

Keywords colloidal carbon dots, wet-chemistry, fluorescence, room temperature phosphorescence, post-purification

\section{Introduction}

Quantum dots (QDs) are semiconducting nanocrystals with sizes less than $20 \mathrm{~nm} .^{[1,2]}$ The optical and electrical properties of QDs can be well-controlled by tuning their sizes/shapes/ dimensions. ${ }^{[3]}$ Compared to inorganic quantum dots (QDs), carbon QDs (C-dots) have attracted a lot of attentions due to their unique properties, ${ }^{[4]}$ such as high quantum yield, ${ }^{[5]}$ good photo- and chemical-stability, ${ }^{[6,7]}$ low-cost, ${ }^{[8,9]}$ low-toxicity ${ }^{[10,11]}$ and easy synthesis with earth-abundant precursors. ${ }^{[12]}$ Because of their excellent properties, ${ }^{[13,14]}$ they have been widely used as building blocks for various types of optoelectronic devices, ${ }^{\text {[15] }}$ such as luminescent solar concentrators, ${ }^{[16,17]}$ light emitting devices and photoelectrochemical devices. ${ }^{[18-22]}$ For example, Wang et al. studied the photocatalytic process of composite materials of $\mathrm{C}$-dots and found that the electron transfer performance of C-dots cannot only improve the catalytic performance of semiconductor photocatalysts, but also improve its stability; ${ }^{[23]}$ Lu et al. described the upconversion photoluminescent properties of colloidal C-dots. The as-prepared C-dots can absorb photons in long wavelengths and emit high energy photons in short wavelengths through up-conversion properties; ${ }^{[24]} \mathrm{Li}$ et al. have demonstrated a facile and economical synthesis of nanostructured branched polyethylenimine/ $/ \mathrm{TiO}_{2} / \mathrm{C}$-dots composites via an electrostatic assembly method, which can promote the transfer of interface electrons, thereby prolonging the carrier life and improving the photocatalytic efficiency. ${ }^{[25]}$

\section{Synthesis Methods of QDs}

During last twenty years, there are many methods, which have been reported for the preparation of colloidal C-dots, ${ }^{[26,27]}$ such as hydrothermal/solvothermal synthesis, ${ }^{[28-32]}$ solid-state vacuum heating, ${ }^{[12]}$ microwave-assisted synthesis, ${ }^{[33-36]}$ and pyrolysis carbonization approach (Figure 1). ${ }^{[37]}$ These wet-chemistry methods are very effective for reproducible synthesis of the colloidal C-dots, because they can control the structure and surface functional groups of the C-dots by controlling the types of precursors, reaction temperature and solvents. ${ }^{[38]}$ For example, Wang et al. used the hydrothermal

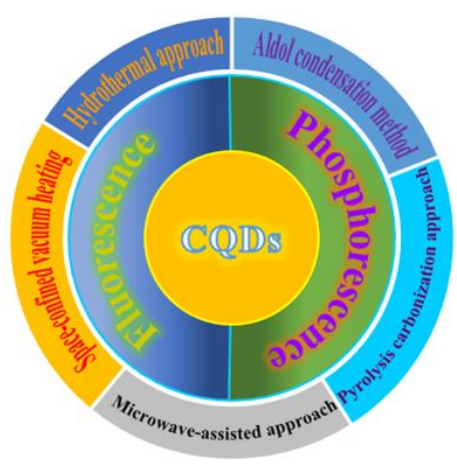

Figure 1 C-dots optical properties and synthesis method.

approach to produce C-dots with different colors, and the quantum yield of red C-dots reaches $\sim 50 \%$, ${ }^{[15]}$ Ye et al. used $p$-phenylenediamine as carbon source and ethanol as solvent to synthesize C-dots. The as-prepared C-dots have strong antibacterial activity ${ }^{[39]} \mathrm{Li}$ et al. proposed a cheap and efficient aldol condensation method to synthesize C-dots at room temperature $;{ }^{[40]}$ Yan et al. used miscanthus plants as carbon precursors to prepare C-dots with good quality and size uniformity via an ultrasound-assisted mechano-chemical cracking method; ${ }^{[41]}$ Yao et al. used a microwave-assisted approach to close-loop condensation of glucose molecules for C-dots production. ${ }^{[42]}$

Although these approaches can produce C-dots, it is still a quite challenge to produce the $\mathrm{C}$-dots with mono-size distribution because of the uncontrolled reaction conditions. For example, it is difficult to monitor the reaction parameter during the hydrothermal and microwave reaction. The wide size distribution leads to the strong overlap between the absorption and emission spectra of as-prepared C-dots, limiting their application in luminescent solar concentrators and light emitting devices; in addition, it is still a big issue for the post-purification of C-dots. ${ }^{[43,44]}$ Typically, the organic precursors and by-products were mixed with $\mathrm{C}$-dots, leading to a gel-like product. Therefore, researchers used the high-speed 

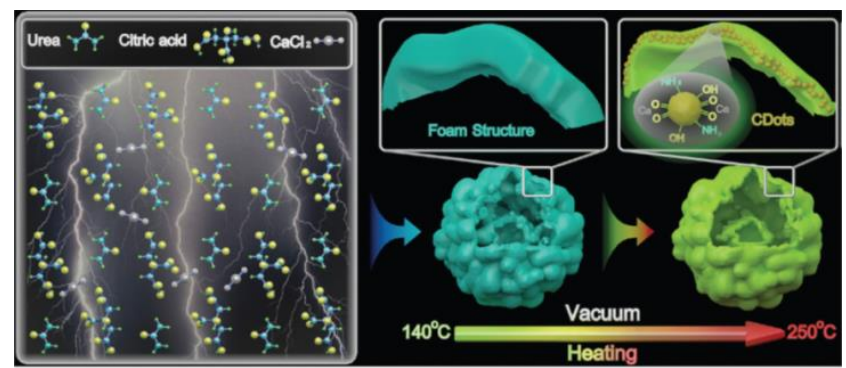

Figure 2 Schematics of the growth mechanism of C-dots synthesized via the vacuum heating method, which occurs through the formation of the blue emissive molecular species at a lower temperature $\left(140{ }^{\circ} \mathrm{C}\right)$, followed by their carbonization and the formation of green-emissive C-dots at higher temperatures $\left(220-250^{\circ} \mathrm{C}\right)$ in the confined-space foam. Reprinted with permission from Nanoscale Horiz. 2019, 4, 388-395. Copyright 2020 Royal Society of Chemistry. ${ }^{[4]}$

centrifugation, silica-gel column chromatography or dialysis to post-purify the product. Then, the freezing-drying approach was used to obtain the C-dots powder. ${ }^{[45,46]}$ This process is complicated and time-consuming. Very recently, Zhou et al. have synthesized high-quality $\mathrm{C}$-dots through a space-confined vacuum heating synthesis using green citric acid and urea as precursors. The presence of the $\mathrm{CaCl}_{2}$ reacts with citric acid, forms an inflated foam under vacuum heating, providing ultrathin spaces of the foam walls for the confined growth of the precursors (Figure 2). The C-dots were grown at $250{ }^{\circ} \mathrm{C}$ under the spatial-confinement, leading to a very narrow size distribution. The C-dots exhibited a high quantum yield of $72 \% .{ }^{[47]}$ Zhao et al. further found that the high quantum yield could be mainly due to the strong interaction between the $\mathrm{Ca}^{2+}$ and $\mathrm{C}=\mathrm{O}$ group, forming a stable energy gap, as proved by the transient absorption spectrum. ${ }^{[48,49]}$ While the quantum yield of the $\mathrm{C}$-dots is sensitive to the $\mathrm{pH}$ value, as the $\mathrm{Ca}^{2+}$ could form the $\mathrm{Ca}(\mathrm{OH})_{2}$ in basic condition. This reaction could break the interaction between the $\mathrm{Ca}^{2+}$ and $\mathrm{C}=\mathrm{O}$, forming the traps, inducing the decrease of the quantum yield. ${ }^{[46]}$

Ren et al. synthesized C-dots using a vacuum heating approach. They found that the $\mathrm{Ca}^{2+}$ has a strong interaction with $\mathrm{C}=\mathrm{O}$ group of the $\mathrm{C}$-dots, contributing the strong absorption at $405 \mathrm{~nm}$, excitation-independent PL behavior and high quantum yield. Removal of $\mathrm{Ca}^{2+}$ by $\mathrm{Na}_{2} \mathrm{CO}_{3}$ led to the disappearance of the absorption at $405 \mathrm{~nm}$ and a decrease of the quantum yield (Figure 3 ). This finding suggests that the surface chemistry is very important for the optical properties of the C-dots. In addition, the selection of the purification method may affect the quantum yield of $\mathrm{C}$-dots.
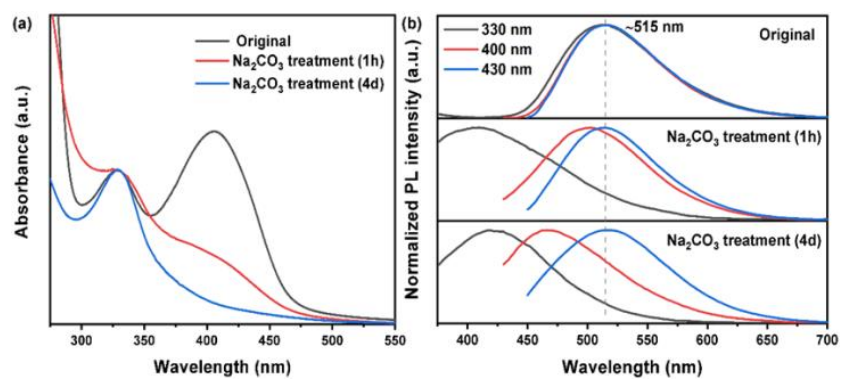

Figure 3 (a) Absorption spectra of C-dots under different treatment conditions. (b) PL spectra of the C-dots treated under different conditions at different excitation wavelengths. ${ }^{[49]}$

Besides the attracted fluorescence in the C-dots, recently room-temperature phosphorescent (RTP) C-dots have been synthesized as they can offer unique opportunities in infor

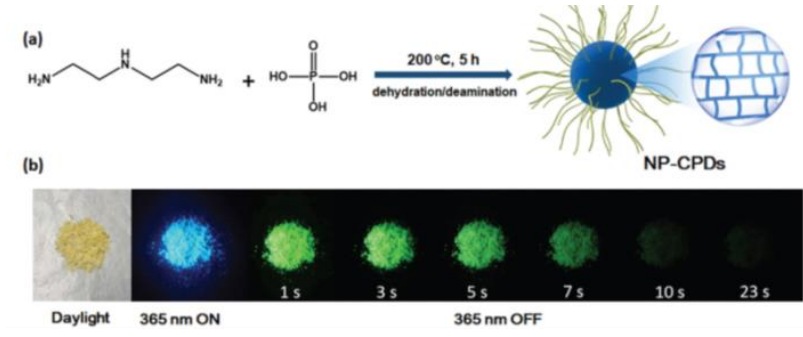

Figure 4 (a) Schematic diagram showing the preparation of NP-CPDs. (b) Photographs of NP-CPD powders captured under daylight and a UV lamp (365 nm) turned ON and OFF from 1 to $23 \mathrm{~s}$, respectively. Reprinted with permission from $\mathrm{J}$. Mater. Chem. C. 2021, 9, 4847-4853. Copyright 2020 Royal Society of Chemistry. ${ }^{[51]}$

mation security and biomedical applications. ${ }^{[50]}$ There are two ways to achieve efficient URTP behavior: (i) introducing heteroatoms, aromatic carbonyl groups into the C-dots to improve intersystem crossing processes between singlet and triplet excited states; (ii) providing a rigid molecular environment to suppress the non-radiative transitions. ${ }^{[4,49]}$ For example, Wang et al. reported a fast processable and one-step synthetic approach to produce $\mathrm{N}$ and $\mathrm{P}$ co-doped C-dots (NP-CPDs). ${ }^{[4]}$ NP-CPDs were fabricated by a fast one-step synthetic process via a hydrothermal approach using diethylenetriamine and phosphoric acid as precursors (Figure 4a). The obtained NP-CPDs appeared as yellow powders with bright blue fluorescence and a quantum yield of $35.6 \%$ under UV lamp (365 $\mathrm{nm}$ ) illumination (Figure 4b). After ceasing the UV illumination, the blue photoluminescence becomes invisible promptly while a green-colored RTP component with QY of $4.5 \%$ emerges (Figure 4b). The NP-CPDs are potentially suitable for preparation of security ink-jet printing. However, it is still a challenge to prepare the RTP C-dots with long lifetime.

\section{Conclusions and Perspectives}

Due to the complicated structure in C-dots, it is still unclear for their detailed structures. In addition, the excitation-dependent optical properties in C-dots need to be investigated deeply. The future research directions may focus on: (i) the investigation of the fluorescence mechanism of the C-dots; (ii) the production of the C-dots with bright red colors; (iii) the simple approaches for the produce the C-dots in commercial large-scale. In addition, as the obtained high-purified C-dots is still very difficult, the future study should solve this issue by finding more efficient and simple post-purification approaches.

\section{Acknowledgement}

We thank the funding supports from the Key Research and Development Program of Shandong Province (Grant No. 2021CXGC011003), the Key Research and Development Program of Binzhou City (Grant No. 2019ZDYF005), the State Key Laboratory of Bio-Fibers and Eco-Textiles (Qingdao University, No. ZKT08), the Shandong Provincial Natural Science Foundation (Grant No. ZR2020ME076), the State Key Laboratory of Bio-Fibers and Eco-Textiles (Qingdao University, No. ZKT18), the Shandong Provincial Natural Science Foundation (Grant No. ZR2020ZD22). We also sincerely thank the team of Professor Guang-Ting Han from the State Key Laboratory of Qingdao University for their discussions and comments.

\section{Author Contributions}

Following are the details of the contributions made by each of the authors for the manuscript: Shi-Huan Ren and Hao Zhou 
collected the literatures and wrote this manuscript; Hai-Guang Zhao and Yuan-Ming Zhang revised and organized this manuscript.

\section{Conflict of Interest}

The authors declare no conflict of interest.

Copyright (C) 2021 Hao Zhou, Shi-Huan Ren, Hai-Guang Zhao and Yuan-Ming Zhang. This article is an open access article distributed under the terms and conditions of the Creative Commons Attribution (CC BY) license (http://creativecommons.org/licenses/by/4.0/). The use, distribution or reproduction in other forums is permitted, provided the original author(s) or licensor are credited and that the original publication in this journal is cited, in accordance with accepted academic practice. No use, distribution or reproduction is permitted which does not comply with these terms.

\section{References}

[1] Li, C.-L.; Ou, C.-M.; Huang, C.-C.; Wu, W.-C.; Chen, Y.-P.; Lin, T.-E.; Ho, L.-C.; Wang, C.-W.; Shi, C.-C.; Zhou, H.-C., Lee, Y. C.; Tzeng, W. F.; Chiou, T. J.; Chu, S.-T.; Cang, J.-S.; Chang, H.-T. Carbon Dots Prepared from Ginger Exhibiting Efficient Inhibition of Human Hepatocellular Carcinoma Cells. J. Mater. Chem. B 2014, 2, 4564-4571.

[2] Li, H.-T.; Kang, Z.-H.; Liu, Y.; Lee, S. T. Carbon Nanodots: Synthesis, Properties and Applications. J. Mater. Chem. 2012, 22, 2423-24253.

[3] Zhou, Y. Q.; Mintz, K. J.; Sharma, S. K.; Leblanc, R. M. Carbon Dots: Diverse Preparation, Application, and Perspective in Surface Chemistry. Langmuir 2019, 35, 9115-9132.

[4] Xu, Q.; Kuang, T. R.; Liu, Y.; Cai, L. L.; Peng, X. F.; Sreenivasan, S. T.; Zhao, P.; Yu, Z.-Q.; Li, N. Heteroatom-Doped Carbon Dots: Synthesis, Characterization, Properties, Photoluminescence Mechanism and Biological Applications. J. Mater. Chem. B 2016, 4, 7204-7219.

[5] Ding, H.; Wei, J.-S.; Zhang, P.; Zhou, Z.-Y.; Gao, Q.-Y.; Xiong, H.-M. Solvent-Controlled Synthesis of Highly Luminescent Carbon Dots with a Wide Color Gamut and Narrowed Emission Peak Widths. Small 2018, 14, e1800612.

[6] Kou, X.-L.; Jiang, S.C.; Park, S. J.; Meng, L.-Y. A Review: Recent Advances in Preparations and Applications of Heteroatom-Doped Carbon Quantum Dots. J. Inorg. Chem., Dalton Trans. 2020, 49, 6915-6938.

[7] Gao, J.; Zhu, M.-M.; Huang, H.; Liu, Y.; Kang, Z.-H. Advances, Challenges and Promises of Carbon Dots. Inorg. Chem. Front. 2017, 4, 1963-1986.

[8] Kang, Z. H.; Lee, S. T. Carbon Dots: Advances in Nanocarbon Applications. Nanoscale 2019, 11, 19214-19224.

[9] Xiao, L.; Sun, H.-D. Novel Properties and Applications of Carbon Nanodots. Nanoscale Horiz. 2018, 3, 565-597.

[10] Lim, S. Y.; Shen, W.; Gao, Z.-Q. Carbon Quantum Dots and their Applications. Chem. Soc. Rev. 2014, 44, 362-381.

[11] Sahu, S.; Behera, B.; Maiti, T. K.; Mohapatra, S. Simple One-Step Synthesis of Highly Luminescent Carbon Dots from Orange Juice: Application as Excellent Bio-Imaging Agents. Chem. Commun. 2012, 48, 8835.

[12] Liu, G.-J.; Wang, X.-H.; Han, G.-T.; Yu, J.-Y.; Zhao, H.-G. Earth Abundant Colloidal Carbon Quantum Dots for Luminescent Solar Concentrators. Adv. Mater. 2020, 1, 119-138.

[13] Peng, Z.-L.; Miyanji, E. H.; Zhou, Y.-Q.; Pardo, J.; Hettiarachchi, S. D.; Li, S.-H.; Blackwelder, P. L.; Skromne, I.; Leblanc, R. M. Carbon Dots: Promising Biomaterials for Bone-Specific Imaging and Drug Delivery. Nanoscale 2017, 9, 17533-17543.

[14] Das, R.; Bandyopadhyay, R.; Pramanik, P. Carbon Quantum Dots from Natural Resource: a Review. Mater. Today Chem. 2018, 8,
96-109.

[15] Wang, X.-H.; Wang, M.-R.; Liu, G.-J.; Zhang, Y.-M.; Han, G.-T.; Vomiero, A.; Zhao, H.-G. Colloidal Carbon Quantum Dots as Light Absorber for Efficient and Stable Ecofriendly Photoelectrochemical Hydrogen Generation. Nano Energy 2021, 86, 106122.

[16] Zhou, Y.-F.; Zhao, H.-G.; Ma, D.-L.; Rosei, F. Harnessing the Properties of Colloidal Quantum Dots in Luminescent Solar Concentrators. Chem. Soc. Rev. 2018, 47, 5589-5866.

[17] Liu, G.-J.; Zhao, H.-G.; Diao, F.-Y.; Ling, Z.-B.; Wang, Y.-Q. Stable Tandem Luminescent Solar Concentrators Based on Cdse/Cds Quantum Dots and Carbon Dots. J. Mater. Chem. C 2018, 6, 10059-10066.

[18] Chung, S.; Revia, R. A.; Zhang, M. Graphene Quantum Dots and Their Applications in Bioimaging, Biosensing, and Therapy. Adv. Mater. 2021, 33, e1904362.

[19] Dai, Q.; Zhao, H.; Cao, H.-B.; Wu, Y.-Q.; Sun, Z.; Meng, X.-F.; Wang, T.-Y.; Yu, G.-F.; Lin, J.-Y.; Hou, R. Green Fabrication of Carbon Dots upon Photoirradiation and Their Application in Cell Imaging. ACS Appl. Nano Mater. 2019, 2, 3404-3413.

[20] Molaei, M. J. Principles, Mechanisms, and Application of Carbon Quantum Dots in Sensors: a Review. Anal. Methods 2020, 12, 1266-1287.

[21] Wang, R.; Lu, K.-Q.; Tang, Z.-R.; Xu, Y.-J. Recent Progress in Carbon Quantum Dots: Synthesis, Properties and Applications in Photocatalysis. J. Mater. Chem. A 2017, 5, 3717-3734.

[22] Tuerhong, M.; Xu, Y.; Yin, X.-B. Review on Carbon Dots and Their Applications. Chin. J. Anal. Chem. 2017, 45, 139-150.

[23] Wang, R.; Lu, K.-Q.; Tang, Z.-R.; Xu, Y.-J. Recent Progress in Carbon Quantum Dots: Synthesis, Properties and Applications in Photocatalysis. J. Mater. Chem. A 2017, 5, 3717-3734.

[24] Lu, K.-Q.; Quan Q.; Zhang, N.; Xu, Y.-J. Multifarious Roles of Carbon Quantum Dots in Heterogeneous Photocatalysis. J. Energy Chem. 2016, 25, 927-935.

[25] Li, S.-H.; Weng, B.; Lu, K.-Q.; Xu, Y.-J. Improving the Efficiency of Carbon Quantum Dots as a Visible Light Photosensitizer by Polyamine Interfacial Modification. Wu Li Hua Xue Xue Bao 2018, 34, 708-718.

[26] Hsu, P. C.; Shih, Z. Y.; Lee, C. H.; Chang, H.-T. Synthesis and Analytical Applications of Photoluminescent Carbon Nanodots. Green Chem. 2012, 14, 917-920.

[27] Dager, A.; Uchida, T.; Maekawa, T.; Tachibana, M. Synthesis and Characterization of Mono-Disperse Carbon Quantum Dots from Fennel Seeds: Photoluminescence Analysis Using Machine Learning. Sci. Rep. 2019, 9, 14004.

[28] Dong, D.; Liu, T.-J.; Liang, D.-P.; Jin, X.-P.; Qi, Z.-H.; Li, A.-F.; Ning, Y. Facile Hydrothermal Synthesis of Chlorella-Derived Environmentally Friendly Fluorescent Carbon Dots for Differentiation of Living and Dead Chlorella. ACS Appl. Bio Mater. 2021, 4, 3697-3705.

[29] Atchudan, R.; Jebakumar, I. E.; Shanmugam, M.; Perumal, S.; Somanathan, T.; Lee, Y. R. Sustainable Synthesis of Carbon Quantum Dots from Banana Peel Waste Using Hydrothermal Process for in Vivo Bioimaging. Physica E Low Dimens. Syst. Nanostruct. 2021, 126, 114417.

[30] Han, Z.; Long, Y.-W.; Pan, S.; Liu, H.; Yang, J.-D.; Hu, X.-L. Efficient One-Pot Synthesis of Carbon Dots as a Fluorescent Probe for the Selective and Sensitive Detection of Rifampicin Based on the Inner Filter Effect. Anal. Methods 2018, 1, 485-493.

[31] Lu, H.-Z.; Xu, S.-F.; Liu, J.-Q. One Pot Generation of Blue and Red Carbon Dots in One Binary Solvent System for Dual Channel Detection of $\mathrm{Cr} 3+$ and $\mathrm{Pb} 2+$ Based on Ion Imprinted Fluorescence Polymers. ACS Sens. 2019, 4, 1917-1924.

[32] Prasannan, A.; Imae, T. One-Pot Synthesis of Fluorescent Carbon Dots from Orange Waste Peels. Ind. Eng. Chem. Res. 2013, 52, 15673-15678.

[33] Rodriguez, P. D.; Algarra, M.; Tarelho L. A.; Frade, J.; Franco, A. Miguel, G.; Jime, N. J; Rodri, C. E.; Luque, R. Catalyzed 
Microwave-Assisted Preparation of Carbon Quantum Dots from Lignocellulosic Residues. ACS Sustain. Chem. Eng. 2018, 6, 7200-7205.

[34] Jiang, K.; Wang, Y.; Gao, X.; Cai, C.; Lin, H. Facile, Quick, and Gram-Scale Synthesis of Ultralong-Lifetime Room-TemperaturePhosphorescent Carbon Dots by Microwave Irradiation. Angew. Chem. Int. Ed. Engl. 2018, 57, 6216-6220.

[35] Feng, J.; Zhao, X.-R.; Bian, W.; Tang, X.-J. Microwave-Assisted Synthesis of Nitrogen-Rich Carbon Dots as Effective Fluorescent Probes for Sensitive Detection of Ag. Mater. Chem. Front. 2019, 3, 2751-2758.

[36] Sharma, A.; Das, J. Small Molecules Derived Carbon Dots: Synthesis and Applications in Sensing, Catalysis, Imaging, and Biomedicine. J. Nanobiotechnol. 2019, 17, 92.

[37] Rub, P. S. A.; Chetty, S. S.; Selvarasu, P.; Vadivel, M. A.; Kumar, Y.; Periyasamy, L.; Santhakumar, M.; Sadras, S. R.; Santhakumar, K. Transition Metal Ion $\left(\mathrm{Mn}^{2+}, \mathrm{Fe}^{2+}, \mathrm{Co}^{2+}\right.$, and $\left.\mathrm{Ni}^{2+}\right)$ Doped Carbon Dots Synthesized via Microwave-Assisted Pyrolysis: A Potential Nanoprobe for Magneto-fluorescent Dual-Modality Bioimaging. ACS Biomater. Sci. Eng. 2018, 4, 2582-2596.

[38] Baig, N.; Kammakakam, I.; Falath, W. Nanomaterials: A Review of Synthesis Methods, Properties, Recent Progress, and Challenges. Adv. Mater. 2021, 2, 1821-1871.

[39] Ye, Z.-G.; Li, G.-X.; Lei, J. One-Step and One-Precursor Hydrothermal Synthesis of Carbon Dots with Superior Antibacterial Activity. ACS Appl. Bio Mater. 2020, 3, 7095-7102.

[40] Li, L.; Li, Y.; Ye, Y.; Guo, R.; Wang, A.; Zou, G.; Hou, H.; Ji, X. Kilogram-Scale Synthesis and Functionalization of Carbon Dots for Superior Electrochemical Potassium Storage. ACS Nano. 2021, 15, 6872-6885

[41] Yan, Y.-X.; Manickam, S.; Lester, E.; Wu, T.; Pang, C.-H. Synthesis of Graphene Oxide and Graphene Quantum Dots from Miscanthus via Ultrasound-Assisted Mechano-Chemical Cracking Method. Ultrason. Sonochem. 2021, 73, 105519.

[42] Yao, M.; Huang, J.; Deng, Z.; Jin, W.; Yuan, Y.; Nie, J.; Wang, H.; Du, F; Zhang, Y. Transforming Glucose into Fluorescent Graphene Quantum Dots via Microwave Radiation for Sensitive Detection of $\mathrm{Al}(3+)$ Ions Based on Aggregation-Induced Enhanced Emission. Analyst 2020, 145, 6981-6986.

[43] Koutsioukis, A.; Akouros, A.; Zboril, R.; Georgakilas, V. Solid Phase Extraction for the Purification of Violet, Blue, Green and
Yellow Emitting Carbon Dots. Nanoscale 2018, 1, 11293-11296.

[44] Zhou, S.-J.; Sui, Y.; Zhu, X.-T.; Sun, X.-F.; Zhuo, S.-P.; Li, H.-G. Study and Comparison on Purification Methods of Multicolor Emission Carbon Dots. Chem.-Asian. J. 2021, 16, 348-354.

[45] Tian, R.-B.; Zhong, S.-T.; Wu, J.; Geng, Y.-L.; Zhou, B.-J.; Wang, Q.-H.; Jiang, W. Facile Preparation and the Stepwise Formation Mechanistic Investigation of Gram-Scale Nitrogen-Doped Graphene Quantum Dots. J. Mater. Chem. C 2017, 5, 9174-9180.

[46] De, S. H. A.; Gupta, S. K.; Mao, Y. On High Purity Fullerenol Obtained by Combined Dialysis and Freeze-Drying Method with its Morphostructural Transition and Photoluminescence. Sep. Purif. Technol. 2019, 210, 927-934.

[47] Zhou, D.; Jing, P.; Wang, Y.; Zhai, Y.; Li, D.; Xiong, Y.; Baranov, A.V.; Qu, S.; Rogach, A.L. Carbon Dots Produced via Space-Confined Vacuum Heating: Maintaining Efficient Luminescence in Both Dispersed and Aggregated States. Nanoscale Horiz. 2019, 4, 388-395.

[48] Zhao, H.-G.; Liu, G.-J; You, S.-J.; Camargo, F. V. A.; Zavelani-Rossi, M.; Wang, X.-H; Sun, C.-C.; Liu, B.; Zhang, Y.-M.; Han, G.-T.; Vomiero, A.; Gong, X. Gram-Scale Synthesis of Carbon Quantum Dots with a Large Stokes Shift for The Fabrication of Eco-Friendly and High-Efficiency Luminescent Solar Concentrators. Energy Environ. Sci. 2021, 14, 396-406.

[49] Ren, S.-H.; Liu, B. X.; Han, G.-T.; Zhao, H.-G.; Zhang, Y.-M. Surface Chemistry in Calcium Capped Carbon Quantum Dots. Nanoscale 2021, 13, 12149-12156.

[50] Wei, X.-Y.; Yang, J.-W.; Hu, L.-L.; Cao, Y.; Lai, J.; Cao, F.-F.; Gu, J.-J.; Cao, X.-F. Recent Advances in Room Temperature Phosphorescent Carbon Dots: Preparation, Mechanism, and Applications. J. Mater. Chem. C 2021, 9, 4425-4443.

[51] Wang, Z.-F.; Shen, J.; Sun, J.-Z.; Xu, B.; Gao, Z.-H.; Wang, X.; Yan, L.-T.; Zhu, C.-F.; Meng, X.-G. Ultralong-Lived Room Temperature Phosphorescence from $\mathrm{N}$ and $\mathrm{P}$ Codoped Self-Protective Carbonized Polymer Dots for Confidential Information Encryption and Decryption. J. Mater. Chem. C 2021, 9, 4847-4853. 